\title{
Interdisciplinary rounds reduced hospital stay and costs and improved staff satisfaction
}

\author{
Curley C, McEachern JE, Speroff T. A firm trial of interdisciplinary rounds on the inpatient medical wards. An intervention \\ designed using continuous quality improvement. Med Care 1998;36:AS4-12.
}

\author{
Question \\ Can interdisciplinary rounds improve efficiency of patient care \\ and staff satisfaction, and decrease costs on inpatient medical \\ units?
}

\section{Design}

Randomised controlled trial.

\section{Setting}

3 inpatient medical units in an acute care, tertiary referral and teaching hospital in Cleveland, Ohio, USA.

\section{Patients}

1102 patients (mean age 53 y, 52\% women) who were admitted to and discharged from the medical unit. Patients who spent $<50 \%$ of their hospital stay on the medical unit or who were transferred to another service were excluded.

\section{Intervention}

On their first admission, patients were allocated to 1 of 3 inpatient medical units from which they received all care for this and any subsequent admissions. Each of the 3 medical units had 2 physician teams. The teams were divided so that both teams from 1 unit and 1 team from another unit conducted daily interdisciplinary rounds, designed by a continuous quality improvement team, and the remaining 3 teams (2 from 1 unit and the remaining team from the other unit) continued traditional rounds. Interdisciplinary rounds included physicians, a nurse patient care coordinator, a pharmacist, a nutritionist, and a social worker; orders were written during rounds with the patient charts present. Traditional daily rounds included only physicians, and interdisciplinary rounds were held once a week; orders were writ- ten throughout the day and patient charts were left at the nursing station. 567 patients were allocated to interdisciplinary rounds and 535 were allocated to traditional rounds.

\section{Main outcome measures}

Length of hospital stay, total charges, hospital death, type of hospital disposition, and healthcare provider satisfaction.

\section{Main results}

Compared with patients who received traditional rounds, those who received interdisciplinary rounds had shorter mean lengths of hospital stay (5.5 $v 6.1 \mathrm{~d}, \mathrm{p}=0.006)$ and lower mean total charges $(\$ 6681 v \$ 8090, \mathrm{p}=0.002)$, but did not differ for rate of hospital death $(1.8 \% v 1.9 \%, \mathrm{p}=0.90)$, patients discharged home $(73.9 \%$ $\% 73.1 \%, \mathrm{p}=0.79)$, and patients discharged to an interim care facility $(9.4 \% v 12.3 \%, p=0.12)$. Factor analysis of satisfaction surveys completed by 21 providers of interdisciplinary rounds and 19 providers of traditional rounds showed that providers of interdisciplinary rounds had a greater understanding of patient care, more effective communication, and more teamwork than providers of traditional rounds ( $\mathrm{p}<0.006$ for each factor)

\section{Conclusion}

Compared with physician focused traditional rounds, interdisciplinary healthcare team rounds led to decreased length of hospital stay, lower costs for patients in hospital on medical units, and improved staff satisfaction.

Source of funding: not stated.

For correspondence: Dr C Curley, Division of General Internal Medicine, Department of Medicine, MetroHealth Medical Center, 2500 MetroHealth Drive, Cleveland OH 44109, USA. Fax +1 216 7785000

A modified version of this abstract appears in ACP Journal Club.

\section{Commentary}

Few controlled trials of interdisciplinary teamwork in health care are available except in geriatric medicine. ${ }^{12}$ The study by Curley $e t$ al is important because of its large sample size, inclusion of financial data, and attention to many factors that can make the relation of care processes and outcomes difficult to demonstrate (eg, patient diagnosis and demographics and severity mix index). Patients in both groups were found to be similar, giving strength to the conclusion that the difference in rounds led to decreased costs and length of stay.

Curley et al used the term "interdisciplinary" to emphasise that the many disciplines did not work independently of one another, but addressed patients' needs within the context of all team members' contributions.
Satisfaction surveys were given only to physicians present at 1 noon conference and to nurses and ancillary staff over a 1 week period. Surveys were completed by 40 providers, primarily nurses, out of $>150$ healthcare workers. Because of these limitations, survey results may not accurately represent perceptions of all providers.

An additional limitation was that "quality of care" data were not available, and study findings could only address aspects of care efficiency. The 2 physician teams were assigned to interdisciplinary or traditional rounds on only 1 of the medical units. Both teams on the second unit conducted interdisciplinary rounds and both teams on the third unit conducted traditional rounds. Differences among units may therefore account for some of the results.
The results are of interest to nurses working on any adult inpatient unit. Benefits to patients, staff, and the organisation might be gained from making 1 or more of the following practice changes: (1) more frequent formal communication among nurses, physicians, and ancillary health professionals; (2) orders written once a day; and (3) orders written during rounds when all team members are present.

Priscilla Sandford Worral, RN, PhD Coordinator, Nursing Research State University of New York Health Sciences Center Syracuse, New York, USA

1. Williams ME, Williams TF, Zimmer JG, et al.J Am Geriatr Soc 1987;35:1071-8. Campion EW, Jette A, Berkman B. J Am Geriatr Soc 1983;31:792-6. 